\title{
Correlation Between the Plasma Cortisol Level and the Characteristics of Magnetic Resonance Spectroscopy of the Prefrontal Cortex and Hippocampus in Depressed Patients
}

\author{
Yi Xu ${ }^{1}$, Zhouli Zheng ${ }^{2}$, Huiyi Deng ${ }^{2}$, Chipeng $\mathrm{Wu}^{2}$, Changzheng Shi ${ }^{3}$, Hao $\mathrm{Xu}^{4, *}$ \\ ${ }^{1}$ Department of Psychology, the First Affiliated Hospital, Jinan University, Guangzhou, China \\ ${ }^{2}$ Department of Public Health and Preventive Medicine the First Affiliated Hospital, Jinan University, Guangzhou, China \\ ${ }^{3}$ Imaging Center, the First Affiliated Hospital, Jinan University, Guangzhou, China \\ ${ }^{4}$ Department of Nuclear Medicine, the First Affiliated Hospital, Jinan University, Guangzhou, China
}

Email address:

txh@jnu.edu.cn (Hao Xu)

${ }^{*}$ Corresponding author

\section{To cite this article:}

Yi Xu, Zhouli Zheng, Huiyi Deng, Chipeng Wu, Changzheng Shi, Hao Xu. Correlation Between the Plasma Cortisol Level and the Characteristics of Magnetic Resonance Spectroscopy of the Prefrontal Cortex and Hippocampus in Depressed Patients. International Journal of Medical Imaging. Vol. 4, No. 5, 2016, pp. 39-43. doi: 10.11648/j.ijmi.20160405.11

Received: July 4, 2016; Accepted: August 18, 2016; Published: September 7, 2016

\begin{abstract}
To explore the characteristic changes of magnetic resonance spectroscopy of prefrontal cortex and hippocampus and the correlation with the plasma cortisol level in depressed patients without any therapy. Subjects were divided into groups by the Hamilton depression scale. Blood was taken to detect the plasma cortisol level. Meanwhile, subjects were scanned by MRI and ${ }^{1} \mathrm{H}-\mathrm{MRS}$ to test the brain structure and the levels of NAA, Cho, $\mathrm{Cr}$ in prefrontal cortex and hippocampus. The level of plasma cortisol, the value of MRS and ${ }^{1} \mathrm{H}-\mathrm{MRS}$ of the patients were compared with those of normal control. We found that the level of plasma cortisol in depression group is higher than that in normal control group $(\mathrm{P}=0.000)$. A negative correlation between the level of plasma cortisol and the level of NAA in the left prefrontal cortex is observed $(r=-0.625, P=0.041)$, while in the left hippocampus, the correlation between them is positive $(r=0.647, \mathrm{P}=0.043)$. The level of NAA in the left prefrontal cortex of depression group is lower than that of normal control $(\mathrm{P}=0.006)$; and the levels of NAA, Cho, $\mathrm{Cr}$ in both left and right hippocampus of depression group is lower than those of normal control group $(\mathrm{P}<0.05)$. These data suggest that the changes of energy metabolism may happen before the structural damage of prefrontal cortex and hippocampus, and correlate to the changes of the level of plasma cortisol in depression patients.
\end{abstract}

Keywords: Depression, Plasma Cortisol, Magnetic Resonance Spectroscopy, Prefrontal Cortex, Hippocampus

\section{Introduction}

Depression is a psychiatric disorder characterized by obvious and long-lasting low mood. So far it is unclear about the cause and the pathomechanism of the disease. Yet it is revealed by the literature review that depression is related to the changes of endocrines, while the hyperfunction of Hypothalamic-Pituitary-Adrenal axis (HPA axis) is the biological characteristic of depression known earlier and studied mostly by human $[1,2]$. As the end product of HPA axis, the plasma cortisol is needed by the body to maintain the physiological function under pressure. However, the continually increased plasma cortisol level will damage prefrontal cortex and hippocampus etc., which are the mood-regulating areas, and then consequently cause symptoms such as depressed mood, cognitive dysfunction and insomnia and so on. However, the correlation between the plasma cortisol level of the depressed patients and the structure and function of such areas hasn't been clear yet. Through analyzing magnetic resonance spectroscopy, this study investigates the structure and function of prefrontal cortex and hippocampus of the depressed patients without any 
therapy, as well as explores their correlations with the plasma cortisol level.

\section{Subjects and Methods}

\subsection{Subjects}

33 depressed patients were collected from the psychiatric wards and clinics of the First Affiliated Hospital of Jinan University from September 2012 to July 2014, in which, 16 are males and 17 are females, with the ages of $28.48 \pm 12.10$. Inclusion criterion: (1) Meeting the diagnostic criteria on depression according to the Diagnostic and Statistical Manual of Mental Disorder-IV, DSM-IV; (2) Age: 15 60; (3) Junior middle school education and above; (4) The score is greater than or equal to 20, according to Hamilton depression scale, HAMD24; and also is greater than or equal to 50, according to self-rating depression scale, SDS; without taking any antidepressant, and for recurrent patients, at least one month after suspending antidepressant therapy; (5) Without taking hormone medicines and contraceptives etc. at least for one month; (6) Right-handedness. Exclusion criteria: (1) Patients with brain organic mental disorders, schizophrenia, or serious physical sicknesses or of abusing alcohol and drugs; (2) Women in menstruation, pregnancy or lactation period; (3) Patients with recent fever, severe aggressive behaviors or mental retardation. The control group is recruited in local community, inclusion criterion: (1) Noncompliance with the diagnostic criteria on any mental disease according to DSM-IV; (2) Age: 15 60; (3) Junior middle school education and above; (4) Score of HAMD2 $4<8$ points and SDA $<40$ points; (5) Score of HAMA $<14$; (6) Physical health, without taking hormone medicines and contraceptives within one month; (7) Right-handedness. The subjects with the contraindication of MRI examination, physical sicknesses or abnormal brain structure by MRI scan are excluded. Total of 20 objects are included in normal control group, in which 15 are males and 5 are females, with the ages of $23.25 \pm 9.47$. And all subjects in this study have signed the informed consent.

\subsection{Score}

The clinical symptoms will be evaluated with HAMD by one attending physician in department of psychiatry. As an internationally-recognized gold standard for evaluating the seriousness of depression (choose HAMD24), HAMD consists of 7 symptom factors according to test contents: anxiety/somatization, change of body mass, cognitive dysfunction, circadian change, retardation, sleep disorder and feeling of despair, which are scored respectively and totally. This study adopts Davis Standard: the total score $>35$ points as severe depression; 20 35 points as mild or moderate depression; and $<8$ points as none.

\subsection{Test for Plasma Cortisol Level}

$4 \mathrm{ml}$ of morning venous blood was taken from all the fasting subjects with vacuum blood collection tube without anticoagulant, and then the centrifugation is performed within
$2 \mathrm{~h}(800 \times \mathrm{g}, 15 \mathrm{~min})$ to separate serum. The ARCHITECH i2000 SR produced by Abbott and supporting reagent kits are used to test. Normal value: $118.6-618 \mathrm{nmol} / \mathrm{L}$.

\subsection{MRI and ${ }^{1}$ H-MRS Examination}

MRI examination adopts Sigma HD1.5T MRI scanner produced by GE Healthcare and 8-channel head and neck phase array extremity coil. The subject's head is usually fixed with sponge-robber pad to avoid motion artifacts, and is put in the accurate position to reach midline and to be bilaterally symmetrical. Axis T1 weighted image (T1W1), T2 weighted image (T2W1) as well as fluid attenuated inversion recovery (FLAIR) scan are performed for all routine examination, and the routine MRI images are diagnosed by two experienced image practitioners. No subjects with obvious morphological abnormalities are founded to enter into the subsequent scan sequence.

\section{5. ${ }^{1} \mathrm{H}$-MRS Examination}

2D ${ }^{1} \mathrm{H}-\mathrm{MRS}$ scan firstly choose T2W1 as scout of MRS, axial scanning, parameters: Time of Repetition $(\mathrm{TR})=5000$, Time of echo $(\mathrm{TE})=113 \mathrm{~m}$, number of excitation $(\mathrm{NEX})=1$, Slice thickness $=5 \mathrm{~mm}$, Gap $=0 \mathrm{~mm}$. The volume of interest (VOI) is determined on axis T1WI image, the VOI of frontal lobe chooses the prefrontal white matter and anterior cingulate cortex of bilateral anterior horn of lateral ventricle, to avoid the influence from lateral ventricle and the cerebrospinal fluid in cerebral sulcus; as for the VOI of hippocampus, the slice taking hippocampus head is chosen as the center, to avoid touching basicranial structure. As for multi-voxel 2D ${ }^{1} \mathrm{H}-\mathrm{MRS}$ examination, the point-resolved selective spectroscopy (PRESS) is adopted, TR $=1000 \mathrm{~ms}, \mathrm{TE}=144 \mathrm{~ms}, \mathrm{NEX}=1$, Field of view $=24 \mathrm{~cm} \times 24 \mathrm{~cm}$, Matrix size $18 \times 18$, scalp fat and air around saturated VOI, full width at half maximum (FWHM) $<10 \mathrm{~Hz}$, water inhibition $>98 \%$, scanning time: 5 $\min 28 \mathrm{~s}$.

After MRS data are subject to imaging analysis and the treatment by GE AW4.2 work station, the color distribution map of varieties of metabolites is obtained, and then overlap metabolite map with MRI scout. The minimum voxel (1 voxel $=56.2 \mathrm{~mm}^{3}$ ) in bilateral frontal cortex and hippocampus are taken as unit of measurement, and the curve of 2D1HMRS of brain tissues in these areas is obtained, and the areas under the peaks of N-acetyl aspartate (NAA), choline (Cho) and creatine $(\mathrm{Cr})$ in each brain area is measured. The obtained images and data are treated by one experienced image practitioner without knowledge of any clinical information of subjects, to eliminate the deviation of sampling location and identify the stability of spectrometry.

\subsection{Statistical Methods}

SPSS19.0 is used to establish the database. T test, ANOVA Analysis and Pearson analysis are adopted to analyze the correlation of age-sex composition, change of plasma cortisol level and function index values of MRS between depressed patients and normal control group. 


\section{Results}

\subsection{Comparison of Age-Sex Composition Between Depression Group and Normal Control Group}

The comparison of $95 \%$ confidence interval of average age of 33 depressed patients (age 24.4 32.6) with that of 20 subjects in normal control group (age 19.1 27.5) and t-test proves that there is no significant difference for the ages between two groups $(\mathrm{t}=1.65, \mathrm{P}=0.105)$. In depression group, there are 16 male and 17 female depressed patients, accounting for $48.5 \%$ and $51.5 \%$ respectively; in normal control group, there are 15 male and 5 female depressed patients, accounting for $75.0 \%$ and $25.0 \%$ respectively; and $\chi^{2}$ examination proves that there is also no significant difference for the sex composition between two groups $\left(\chi^{2}=3.61, \mathrm{P}=0.058\right)$.

\subsection{Change of Plasma Cortisol Level of Depressed Patients}

According to Hamilton Depression Scale scores, 33 depressed patients are divided into two groups. In one group, 16 patients with scores less than 35 points are regarded as moderate depression; in the other group, 17 patients with scores greater than 35 points are regarded as severe depression. The comparison of plasma cortisol level of moderate and severe depression patients with those of normal control group by ANOVA analysis method shows the level of plasma cortisol of depressed patients is higher than that of normal person. As the depression aggravates, the level of plasma cortisol of depressed patients also increases. The level of plasma cortisol of severe depression patients is higher than that in normal control group, and there is statistical significant difference $(\mathrm{F}=21.049, \mathrm{P}=0.000$, Table 1$)$.

Table 1. The comparison of plasma cortisol level of depressed patients with that of normal control group $(\overline{\boldsymbol{x}} \pm \boldsymbol{s})$.

\begin{tabular}{lllll}
\hline Depression level & $\boldsymbol{n}$ & $\begin{array}{c}\text { plasma cortisol } \\
\text { level (nmol/L) }\end{array}$ & $\boldsymbol{F}$ & $\boldsymbol{P}$ \\
\hline Normal control group & 20 & $272.43 \pm 154.28$ & 21.049 & 0.000 \\
Moderate depression group & 16 & $380.49 \pm 190.87$ & & \\
Severe depression group & 17 & $652.50 \pm 199.46$ & & \\
\hline
\end{tabular}

\subsection{MRI and Comparison of ${ }^{1} H$-MRS Indexes in Prefrontal Cortex and Hippocampus}

The depressed patients are scanned by using MRI to test brain structure, glucose metabolism condition are compared with those of normal control group. The results show that no obvious organic changes are observed in brain tissues of all depressed patients. However, both the level of NAA in the left prefrontal cortex of depression group and the levels of NAA, Cho, $\mathrm{Cr}$ in both left and right hippocampus of depression group are lower than those of normal control group. And the ANOVA analysis results show that there is significant difference. But the levels of NAA, Cho, $\mathrm{Cr}$ in both left and right hippocampus of all severe depression patients increase to some extent, and are higher than those of moderate depression patients, while the LSD test of multiple comparison shows that there is significant difference only in the $\mathrm{Cr}$ level in left hippocampus (Table 2).

Table 2. Comparison of ${ }^{l} H-M R S$ indexes in prefrontal cortex and hippocampus of depressed patients with those of normal control group ( $\overline{\boldsymbol{x}} \pm \mathbf{s}$ ).

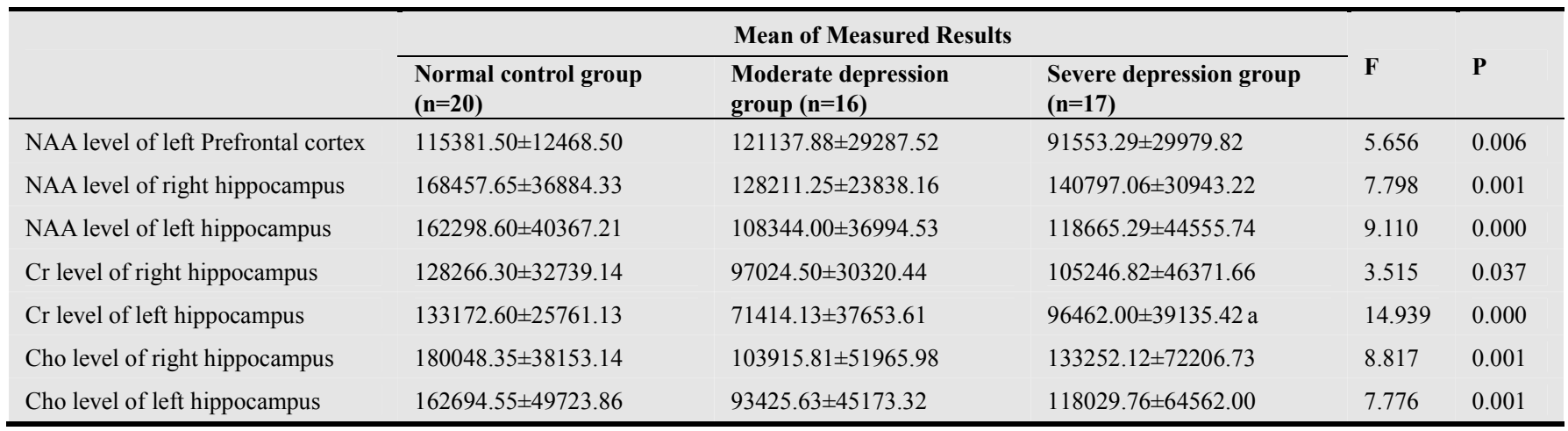

a denotes that the severe depression group has statistically significant difference with moderate depression group $(\mathrm{P}<0.05)$.

\subsection{The Characteristic Changes of Magnetic Resonance Spectroscopy of Prefrontal Cortex and Hippocampus and Its Correlation with the Plasma Cortisol Level in Depressed Patients}

The partial correlative analysis is performed for the plasma cortisol level of 33 depressed patients and each measured value of their MRS [4], in the process of analysis, patients' age and sex are controlled as confounding factors. Results show that in the depression group, there is a negative correlation between the plasma cortisol level and the NAA level in the left prefrontal cortex $(\mathrm{r}=-0.625, \mathrm{P}=0.041)$, while there is a positive correlation between the plasma cortisol level and the
NAA level in the left hippocampus $(\mathrm{r}=0.647, \mathrm{P}=0.043)$. The data reveal that when the plasma cortisol level increases, the NAA level in the left prefrontal cortex will decrease and the NAA level in the left hippocampus will increase.

\section{Discussion}

For a long time, hypothalamus-pituitary-adrenal axis (HPA axis) is the neuroendocrine axis mostly studied in pathogenic mechanism of depression. Under emergency state, human body will promote adrenal to excrete cortisol by HPA axis, but the increase of cortisol level may cause apoptosis of hippocampal neurons, and consequently result in depression 
[5]. Our results shows that the plasma cortisol level in depression group is higher than that in normal control group. As depression aggravates, the plasma cortisol level of depressed patients also increases, the plasma cortisol level of severe depression patients is higher than that in moderate depression group and this is statistically significant difference. Many studies have founded the volumetric reduction of bilateral hippocampus of depressed patients [7], but, in this study, no obvious organic changes are found in routine MRI examination. The study conducted by Tadafumi etc., also found that the change of NAA level observed by MRS happened before the morphological changes in brain observed by CT or MRI [8]. As the derivative of aspartic acid, the intermediate of tricarboxylic acid cycle [9], NAA mainly exists in neurons, and the decrease of NAA level may indicate the structure damage and dysfunction of neurons. Cho reflects the total choline content, which is an important precursor substance for neurotransmitter acetylcholine, membrane phospholipid, phosphatidylcholine and sphingomyelin and serves as the biological marker of membrane phospholipid metabolism in cell, reflecting cell membrane function [12]. Cr serves as the storage form of high-energy phosphates and the buffer of ATP and ADP. When more ATP is needed to supply energy with the enhancing of metabolism of brain tissue, phosphocreatine can be converted into $\mathrm{Cr}$ under creatine phosphokinase. That is, when metabolism of brain tissue enhances, more $\mathrm{Cr}$ will be produced while consuming ATP [10]. Therefore, the reduction of $\mathrm{Cr}$ concentration indicates the slowdown of metabolism of brain tissue. The MRS result of this study shows the NAA level in the left prefrontal cortex of depressed patients is lower than that of normal control, the difference is significant, and as the depression aggravates, the decrease of NAA level is more obvious, which is consistent with previous reports by Soeri and Gonul $[11,12]$. In addition, the levels of NAA, Cho, Cr in both left and right hippocampus of depression group is lower than those of normal control group, however, the levels of NAA, Cho, $\mathrm{Cr}$ in both left and right hippocampus of all severe depression patients increases to some extent and is higher than those of moderate depression patients, in which the increase of $\mathrm{Cr}$ value is most obvious and there is statistically significant difference. This indicates that as the depression aggravates, the glucose metabolism in hippocampus tends to be active.

This study also analyses the correlation between the plasma cortisol level and different metabolites in prefrontal cortex and hippocampus, and the result shows there is a negative correlation between the plasma cortisol level of depressed patients and the NAA level in the left prefrontal cortex is observed; when the plasma cortisol level of depressed patients increases, the NAA level in the left prefrontal cortex will decrease, the increase of cortisol level may cause the decrease of density and function of neurons in the left prefrontal cortex, and then consequently cause specific behavioral and cognitive dysfunction. The correlation between cortisol level and the NAA level in hippocampus is complicated. Some studies think the decrease of NAA level changes the afferent signals from hippocampus to hypothalamus, and consequently influences the function of HPA axis and further leads to the change of cortisol level [13]. Compared with the normal control group, the NAA level in hippocampus of depressed patients is lower, but for depressed patients, the NAA level in the left hippocampus also increases with the increase of cortisol level. The correlation between them is positive, which indicates that when the cortisol level increases to a certain point, it may facilitate the hippocampus cell metabolism to some extent, and this may correlate with the body's self-regulation mechanism and need to be further explored.

\section{Conclusion}

The changes of energy metabolism may happen before the structural damage of prefrontal cortex and hippocampus, and correlate to the changes of the level of plasma cortisol in depression patients.

\section{References}

[1] Krishnan KR, Godde KM, Kim YD, et al. Psychoneuroendocrinology and brain imaging in depression. Psychiatr Clin North AM, 1998; 21 (2): 465-72.

[2] Nemeroff CB, Vale WW. The neurobiology of depression: inroads to treatment and new drug discovery. J Clin Psychiatry, 2005; 66 Suppl 7: 5-13.

[3] Herman JP, Ostrander MM, Mueller NK et al. Limbic system mechanisms of stress regulation: hypothalano-pituitary-adrenocortical axis. Prog Neupsychopharmacol Biol Psychiatry, 2005; 29 (8): 1201-13.

[4] Yan Likun Application of Correlation Coefficient and Biased Correlation Coefficient in Related Analysis. Journal of Yunnan University of Finance and Economics, 2003, 19 (3): 78-80.

[5] Hoschl C, Hajek. Hippocampal damage mediated by corticosteroids - a neuropsychiatric research challenge. Eur Arch Psychiatry Clin Neurosci, 2001; 251 Suppl 2: 1181-8.

[6] Vreeburg SA, Hoogendijk WJ, Van Pelt J, et al. Major depressive disorder and hypothalamic-pituitary-adrenal axis activity: results from a large cohort study. Arch Gen Psychiatry, 2009; 66 (6): 617-26.

[7] Cole J, Costafreda SG, McGuffin P, et al. Hippocampal atrophy in first episode depression: a meta-analysis of magnetic resonance imaging studies. J Affect Disord, 2011; 134 (1-3): 483-7.

[8] Tadafumi K, Toshiro I, Nobumasa K, et al. Magnetic resonance spectroscopy in affective disorders. J Neuropsychiatry Clin Neurosci, 1998, 10 (2): 133-147.

[9] Tsai G, Coyle JT. N - acetylaspartate in neuropsychiatric disorders. Porg Neurobiol, 1995, 46: 531-540.

[10] Liu Xinguang, Luo Desheng. Biochemistry, Beijing Science Press, 2007.

[11] Sozeri-Varma G, Kalkan - Oquzhanoqlu N, Efe M, et al. Neurochemical metabolites in prefrontal cortex in patients with mild/moderate levels in first-episode depression. Neuropsychiatr Dis Treat, 2013, 9: 1053-9. 
[12] Gruber S, Frey R, Mlynarik V et al. Quantification of metabolic differences in the frontal brain of depressive patients and controls obtained by ${ }^{1} \mathrm{H}-\mathrm{MRS}$ at 3 Tesla. Invest Radiol, 2003; 38 (7): 403-8.
[13] Jacobson L, Sapolsky R. The role of the hippocampus in feedback regulation of the hypothalamic - pituitary adrenocortical axis. Endocrine reviews, 1991; 12 (2): 118-34. 